\title{
Nonlinear model predictive control of a wave energy converter based on differential flatness parametrization
}

\author{
Guang $\mathrm{Li}^{\mathrm{a}}$ \\ ${ }^{a}$ School of Engineering and Material Sciences Queen Mary, University of London, Mile End Road, London E1 4NS, United \\ Kingdom
}

\begin{abstract}
This paper presents a fast constrained optimization approach, which is tailored for nonlinear model predictive control of wave energy converters (WEC). The advantage of this approach relies on its exploitation of the differential flatness of the WEC model. This can reduce the dimension of the resulting nonlinear programming problem (NLP) derived from the continuous constrained optimal control of WEC using pseudospectral method. The alleviation of computational burden using this approach helps to promote an economic implementation of nonlinear model predictive control strategy for WEC control problems. The method is applicable to nonlinear WEC models, nonconvex objective functions and nonlinear constraints, which are commonly encountered in WEC control problems. Numerical simulations demonstrate the efficacy of this approach.
\end{abstract}

Keywords: wave energy, nonlinear model predictive control, differential flatness, pseudospectral method

\section{Introduction}

Sea wave energy extraction is an immature yet promising technology that has received increasing interests from control communities in recent years. The associated control problem is widely acknowledged as challenging due to the unconventional control requirements and the harsh sea conditions, etc.

Sea wave energy converters (WECs) are the devices used to harness wave energy. The control objective of a WEC is to extract the maximum time average power and reduce the risk of device damage, so that the unit cost of the generated electricity can be decreased. Conventional WEC control methods are mainly based on the principle that the maximum energy output is reached when the resonant frequency of the WEC matches the dominant frequency of the incoming waves. These include the impedance matching method by tuning the dynamic parameters of the devices [1-4], the latching control by locking the body at some moments to keep its oscillation in phase with the excitation force [5-10], and the declutching control by declutching the float from the generator at appropriate moments for the same reason. These control methods were developed in idealized cases for monochromatic wave excitations and inactive physical constraints, and can become too complicated to be implemented in realistic scenarios.

Recent works show that the WEC control can be formulated as a constrained optimal control problem: maximizing the energy output subject to physical constraints due to safety considerations. The constrained optimal control of WECs is a non-causal control problem, which needs to be combined with a real-time wave prediction algorithm. This necessitates the online implementation of the optimization problem using the model predictive control (MPC) strategy to calculate a optimal control trajectory at each instant subject to the changing wave profile. The effectiveness of the MPC of WEC has been confirmed under different scenarios by numerical simulations, e.g. [11-14]. However, the computational burden for solving the constrained optimization of WEC control problem can be heavy enough to invalidate the online implementation of MPC

Email address: g.li@qmul.ac.uk (Guang Li) 
scheme economically. The computational difficulties are mainly from the following factors. First, different from the conventional MPC for tracking or regulation problems, the optimization objective of WEC control is to maximize the energy output, which normally results in a nonconvex optimization problem [14-16]. Second, the WEC model can be nonlinear due to the WEC designs, nonlinear power take off (PTO) and the significant nonlinear hydrodynamic effects caused by large incoming waves. Furthermore, the constraints imposed on the WEC control can also be nonlinear and nonconvex, e.g., the constraint for one directional power transfer from the WEC to the grid is nonlinear. All these invalidate the direct implementation of the MPC using the mature convex quadratic programming (QP) optimization algorithms.

To cope with the computational problems associated with the MPC of WECs, different optimization methods have been proposed. In [14], the constrained optimization problem is solved by forward dynamic programming (FDP), which can handle nonlinear models and nonconvex optimization problems; however, the computational speed is only acceptable for low order model. In [15], a modified objective function is proposed, which leads to a convex QP; this method is efficient for dealing with high order linear model and is extended to MPC of an array of WECs [17], but it cannot cope with nonlinear constraints and nonlinear models. Nonlinear MPC (NMPC) of a WEC based on discretization of the nonlinear model is reported in $[18,19]$. But the computational burden can be heavy for online implementation.

In this paper, a computationally efficient optimization approach is proposed for online implementation of NMPC on WECs. The advantages of applying this approach in WEC control are reflected in three aspects:

i) It can directly cope with the nonconvex objective function originally formulated from WEC energy extraction, which is different from other approaches based on convex optimization of approximated objective functions, e.g. $[13,15]$.

ii) It can tackle nonlinear WEC models and nonlinear constraints effectively. Nonlinearities can be commonly encountered in WEC dynamics in many scenarios. Nonlinear constraints can also be present in some cases, e.g. unidirectional power delivery.

iii) It has remarkable computational efficiency even for a high order model, which facilitates its online implementation using economic computational hardware.

The advantages of this approach can be accredited to the combination of two techniques: pseudospectral method and differential flatness, which are specifically tailored for the WEC control problem. Originally developed for numerical solutions of differential equations, spectral method or pseudospectral method has become an efficient tool to solve optimal control problems [20-23]. This method transforms a continuous optimization problem to a nonlinear programming problem (NLP), so that well-developed optimization algorithms, such as sequential quadratic programming, interior-point method and active-set method, can be applied directly. Here we use this optimization method in an MPC framework to generate the optimal control sequence. The advantage of using pseudospectral method is that it provides a direct optimization approach to cope with nonlinearities, nonconvex objective functions and nonlinear constraints in an efficient way. Pseudospectral method is used in [24] for offline WEC design, which is not intended for online implementation.

To further expedite the computational speed for online implementation purpose, this paper exploits the differential flatness property of the WEC model, which is used to reduce the dimension of the NLP. The concept of differential flatness can be regarded as an extension of the controllability of a linear system to that of the nonlinear system [25], and it has been used in optimization, trajectory planning and control design, e.g. [26-30]. Using flatness property, only one variable, called flat output, needs to be parameterized, while all the other state and input variables can be represented by functions of the flat output and its derivatives. The use of the least number of parameterized variables can significantly reduce the computational time.

To show the usage and efficacy of this approach, this paper uses the control of a typical type of a point absorber as a demonstration example, shown in Fig. 1, which roughly corresponds to the power buoy PB150, designed by OPT Inc. A point absorber's horizontal dimension is much less than a nominal wave length. The current researches on WEC control based on MPC are mainly for this type of device. A nonlinear model is used to describe the dynamics of this device. The nonlinearities of a WEC model can come from 


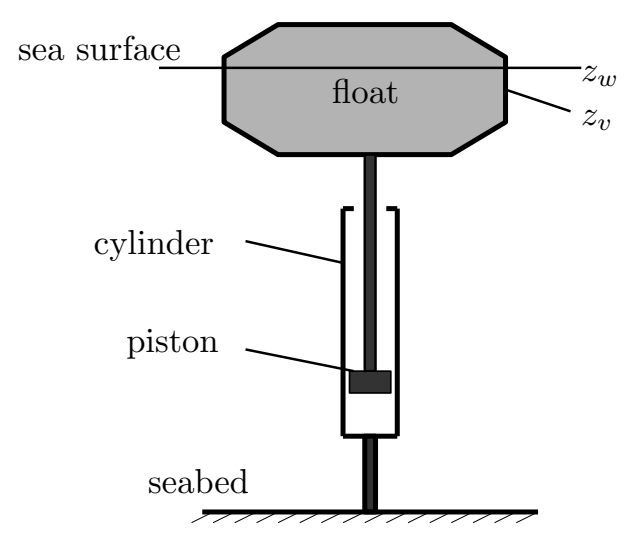

Figure 1: Schematic diagram of the point absorber

different sources, such as hydrodynamic wave-body interaction, the mechanical design, PTO mechanism, and the mooring cables, etc. Here we are assuming that the major nonlinear effect is from the buoyancy force due to the nonconstant radius of the cylindrical float, which is a typical design of the PB150. We use this nonlinearity mainly for the purpose of demonstrating the efficacy of the proposed approach and it can be extended for coping with other types of nonlinearities associated with WECs. Although the focus of this paper is not on the influence of the nonlinearities on the WEC performance, the method can be used as an efficient tool for the investigations of the nonlinearity effects related to WEC design and control.

The structure of the paper is as follows. The WEC modeling and control problem is set up in section 2. The optimization based on psuedospectral method and the flatness is presented in section 3 . Numerical simulation result is shown in section 4 and the paper is concluded in section 5 .

\section{WEC model and control}

The point absorber is shown in Fig. 1. Its modelling and constrained optimal control for a linear model representation have been presented in our earlier work $[14,15]$. In this section, we first briefly describe this device then establish its nonlinear model.

On the sea surface is a float, which is a cylinder with a variational radius, which is different from the assumption of a constant radius cylinder in our previous works [14, 15]. Below the float is a vertically installed hydraulic cylinder. The cylinder is attached at the bottom to the seabed, or an anti-heave plate which is assumed motionless compared with the float, as the design of PB150. The heave motion of the float drives the pistons inside the hydraulic cylinders to produce a liquid flow, which drives a hydraulic motor attached to a synchronous generator. From here, the power reaches the grid via back-to-back AC/DC/AC converters; see [31] for more details related to the power electronics. Here $z_{w}$ is the water level, $z_{v}$ is the height of the mid-point of the float. The control input is the $q$-axis current in the generator-side power converter, to control the electric torque of the generator [32]. The generator torque is proportional to the force $f_{u}$ acting on the pistons from the fluid in the cylinder. Since the motion of the float imposes a velocity $v=\dot{z}_{v}$ on the piston, the extracted power $P(t)$ at time $t$ is expressed as

$$
P=-f_{u} v
$$

The extracted energy over a period $[0, T]$ is therefore

$$
-\int_{0}^{T} f_{u} v d t
$$

For safety considerations, two constraints have to be considered in a WEC. One concerns the relative motion of the float to the sea surface (it should neither sink nor raise above the water and then slam), which 


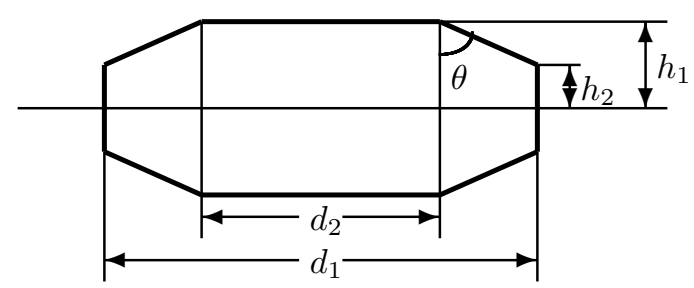

Figure 2: The schematic diagram of the float for the calculation of nonlinear hydraulic stiffness.

can be expressed as

$$
\left|z_{w}-z_{v}\right| \leq z_{\max }
$$

The other constraint is on the control signal set by limitations on the allowable converter current. This constraint can be expressed as

$$
\left|f_{u}\right| \leq u_{\max }
$$

The control objective is to maximize the extracted energy subject to the constraints (3) and (4).

The governing equation for the float is

$$
m_{s} \ddot{z}_{v}=f_{s}-f_{r}-f_{f}+f_{u}
$$

where $m_{s}$ is the mass of the float; $f_{s}$ is the buoyancy force; the radiation force is

$$
f_{r}=m_{\infty} \ddot{z}_{v}+\int_{-\infty}^{t} h_{r}(\tau)\left[\dot{z}_{v}(t-\tau)-\dot{z}_{w}(t-\tau)\right] d \tau
$$

where $m_{\infty}$ is the added mass dependent on frequency, but here it is approximated by its value at the infinite frequency; the friction force is

$$
f_{f}=D_{f} \dot{z}_{v}
$$

with $D_{f}$ as the friction coefficient. $f_{u}$ is the control force. The buoyancy force is a nonlinear function of the relative difference between the heave of the float and the water level. The nonlinearity considered is caused by the nonconstant cross sectional radius of the float. This nonlinearity can be calculated according to Fig. 2 , where it is assumed that the draught of the float in static water is at the medium of the height of the float without loss of generality. By straightforward calculation, this nonlinear force can be derived as

$$
f_{s}=\kappa(z)= \begin{cases}\kappa_{0} h_{2}+\kappa_{n}(z), & \text { if } h_{2}<z \leq h_{1} \\ \kappa_{0} z, & \text { if }|z| \leq h_{2} \\ -\kappa_{0} h_{2}+\kappa_{n}(-z), & \text { if }-h_{1} \leq z<-h_{2}\end{cases}
$$

where $z:=z_{w}-z_{v}, \kappa_{0}:=\rho g h_{1}$, with $\rho$ as the density of sea water, $g$ as standard gravity and

$$
\kappa_{n}(z)=\pi g \rho\left[\frac{(\tan \theta)^{2}}{4}\left(z-h_{2}\right)^{4}-\frac{d_{1}}{3} \tan \theta\left(z-h_{2}\right)^{3}+\frac{d_{1}^{2}}{8}\left(z-h_{2}\right)^{2}\right]
$$

with

$$
\tan \theta=\frac{d_{1}-d_{2}}{2\left(h_{1}-h_{2}\right)}
$$

See Fig. 2 for notation meaning of $d_{1}, d_{2}, h_{1}, h_{2}$ and $\theta$, and Fig. 3 for the plot of the buoyancy force when $h_{1}=1.2 \mathrm{~m}, h_{2}=0.6 \mathrm{~m}$. Note that a simplified expression is used in [14].

Substituting (8), (6) and (7) into (5) gives

$$
m_{s} \ddot{z}_{v}=\kappa(z)-m_{\infty} \ddot{z}_{v}-\int_{-\infty}^{t} h_{r}(\tau)\left[\dot{z}_{v}(t-\tau)-\dot{z}_{w}(t-\tau)\right] d \tau-D_{f} \dot{z}_{v}+f_{u}
$$




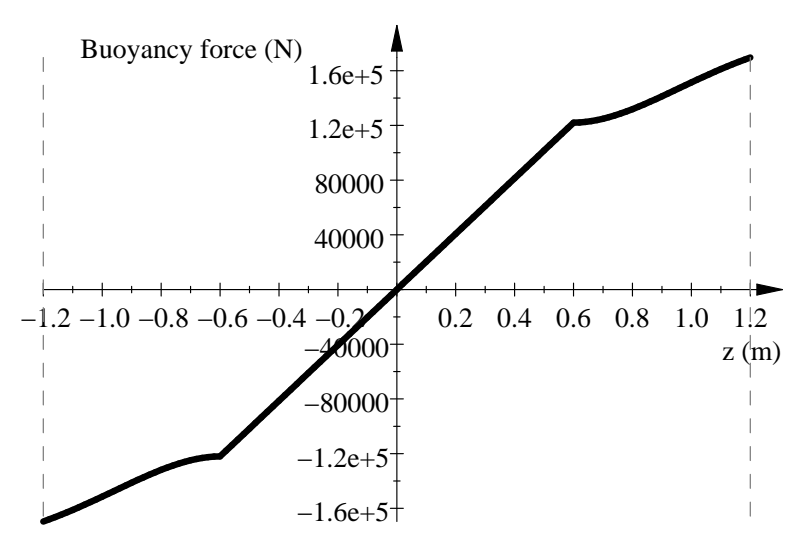

Figure 3: Nonlinear buoyancy force $\left(h_{1}=1.2 \mathrm{~m}, h_{2}=0.6 \mathrm{~m}\right)$.

Here the convolution kernel $h_{r}(\tau)$ can be calculated using commercial software packages for fluid dynamics, and the convolution term can be represented by a state space model

$$
\begin{aligned}
\dot{x}_{r} & =A_{r} x_{r}+B_{r}\left(\dot{z}_{v}-\dot{z}_{w}\right) \\
y_{r} & =C_{r} x_{r}
\end{aligned}
$$

such that $y_{r}:=\int_{-\infty}^{t} h_{r}(\tau)\left[\dot{z}_{v}(t-\tau)-\dot{z}_{w}(t-\tau)\right] d \tau$.

If we choose the state vector as $x=\left[x_{1}, x_{2}, x_{r}\right]^{T} \in \mathbb{R}^{n}$, then the state space model is

$$
\begin{aligned}
\dot{x}(t) & =A x(t)+B_{u} u(t)+B_{w} w(t) \\
y(t) & =C_{y} x(t) \\
z(t) & =C_{z} x(t)
\end{aligned}
$$

with $x_{1}:=z_{w}-z_{v}, x_{2}:=\dot{z}_{v}, u:=f_{u}, w:=\dot{z}_{w}, y:=\dot{z}_{v}$ and

$$
\begin{aligned}
& A=\left[\begin{array}{ccc}
0 & -1 & \mathbf{0}_{1 \times n_{r}} \\
\kappa(\cdot) / m & -D_{f} / m & -C_{r} / m \\
\mathbf{0}_{n_{r} \times 1} & B_{r} & A_{r}
\end{array}\right] \\
& B_{u}=\left[\begin{array}{c}
0 \\
1 / m \\
0
\end{array}\right] \\
& B_{w}=\left[\begin{array}{c}
1 \\
0 \\
-B_{r}
\end{array}\right] \\
& C_{y}=\left[\begin{array}{lll}
0 & 1 & \mathbf{0}_{1 \times n_{r}}
\end{array}\right] \\
& C_{z}=\left[\begin{array}{lll}
1 & 0 & \mathbf{0}_{1 \times n_{r}}
\end{array}\right]
\end{aligned}
$$

Note that (11) describes a nonlinear WEC model due to the presence of the nonlinearity $\kappa(z)$ in matrix $A$. If the radius of the float keeps constant, then (11) becomes a linear model, c.f. [15].

\section{Pseudospectral method and flatness}

The MPC strategy for WEC is to resolve online the following optimization problem formulated from current time $t_{0}$ to the future time $t_{f}$ :

$$
\begin{array}{ll} 
& \min _{\substack{u(t) \\
t \in\left[t_{0}, t_{f}\right]}} \int_{t_{0}}^{t_{f}} u(t) y(t) \\
\text { s.t. } & \dot{x}(t)=A x(t)+B_{u} u(t)+B_{w} w(t) \\
& |z(t)| \leq z_{\max }, \quad|u(t)| \leq u_{\max }, \quad \forall t \in\left[t_{0}, t_{f}\right]
\end{array}
$$

where the wave profile information $w(t)$ needs to be predicted at time $t_{0}$ for the period $\left[t_{0}, t_{f}\right]$ using some wave prediction algorithm, such as deterministic sea wave prediction (DSWP) algorithm [33]. The solution 
of (12) is the optimal control trajectory $\mathbf{u}^{*}(t)$. The control input is the optimal control input at $t_{0}$, i.e. $\mathbf{u}^{*}\left(t_{0}\right)$. In some existing MPC strategies developed for WEC control, the optimization problem (12) is discretized at a fixed sampling time in $\left[t_{0}, t_{f}\right]$, which is then approximated by a QP, see e.g. [13, 15]. In this paper, the optimization problem (12) is parameterized by polynomials based on the pseudospectral method to result in a NLP. This is a direct optimization method, which has been adopted to resolve optimal control problems, and recently applied to WEC optimal design [24]. However, the computational load of implementing this direct optimization method online can be demanding especially for a high order model. To increase the computational efficiency while not losing the solution accuracy, we employ the concept of flatness by exploiting the structure of the WEC model in this section.

\subsection{Flat system}

A nonlinear dynamic system $\dot{x}=f(x, u)$ with $x \in \mathbb{R}^{n}$ and $u \in \mathbb{R}^{m}$ is differentially flat if

(i) there exists a flat output $\xi \in \mathbb{R}^{m}$ which can be represented by a function of the state $x$

$$
\xi=\eta\left(x, u, \dot{u}, \ldots, u^{(a)}\right)
$$

with the mapping $\eta: \mathbb{R}^{n+(a+1) m} \mapsto \mathbb{R}^{m}$, and

(ii) the state $x$ and input $u$ can be expressed in terms of the flat output $\xi$ and a finite number of its derivatives

$$
\begin{aligned}
& x=\varphi_{x}\left(\xi, \dot{\xi}, \ldots, \xi^{(b-1)}\right) \\
& u=\varphi_{u}\left(\xi, \dot{\xi}, \ldots, \xi^{(b)}\right)
\end{aligned}
$$

where the mappings $\varphi_{x}: \mathbb{R}^{b m} \mapsto \mathbb{R}^{n}$ and $\varphi_{u}: \mathbb{R}^{(b+1) m} \mapsto \mathbb{R}^{m}$.

Exploiting the dynamic structure of a flat system can significantly reduce the dimension of the optimization problem converted from the direct method and thus expedite the computational speed. Instead of parameterizing the trajectories along each dimension of $x$ and $u$, we only parameterize the trajectory of the flat output $z$, while the trajectories of $x$ and $u$ can be derived by combinations of a limited number of derivatives of the flat output through (14). When using this method on WEC optimal control problem, the predicted wave trajectory, treated as a measured disturbance, needs to be incorporated. This is distinguished from the existing flatness based optimization methods. In the remaining section, we present the procedure of using the flatness property together with pseudospectral method to convert (12) to a low dimension NLP.

\subsection{WEC optimal control based on flatness}

In the pseudospectral method the numerical integration based on Gauss quadrature interpolation holds for the interval $[-1,1]$. Thus the following relation is needed to transform integral approximations between $t \in\left[t_{0}, t_{f}\right]$ and $\tau \in[-1,1]$

$$
t=\frac{\left(t_{f}-t_{0}\right) \tau+\left(t_{f}+t_{0}\right)}{2}
$$

Suppose the state space realization $\left(A_{r}, B_{r}, C_{r}\right)$ of $(10)$ is in controllable canonical form, such that

$$
\begin{aligned}
\dot{x}_{r, i} & =x_{r, i+1} \text { with } i=1, \ldots, n_{r}-1, \\
\dot{x}_{r, n_{r}} & =\sum_{i=1}^{n_{r}} \alpha_{i} x_{r, i}+x_{2}-w \\
y_{r} & =\sum_{i=1}^{n_{r}} \beta_{i} x_{r, i}
\end{aligned}
$$


where $x_{r}:=\left[x_{r, 1}, x_{r, 2}, \ldots, x_{r, n_{r}}\right]^{T}$. Then a linear transformation of (11) from $t \in\left[t_{0}, t_{f}\right]$ to $\tau \in[-1,1]$ using (15) gives

$$
\begin{aligned}
T_{e} \dot{x}_{1}(\tau) & =-x_{2}(\tau)+w(\tau) \\
T_{e} \dot{x}_{2}(\tau) & =\frac{\kappa\left(x_{1}(\tau)\right)}{m}-\frac{D_{f}}{m} x_{2}(\tau)-\frac{1}{m} \sum_{i=1}^{n_{r}} \beta_{i} x_{r, i}(\tau)+\frac{1}{m} u(\tau) \\
T_{e} \dot{x}_{r, i}(\tau) & =x_{r, i+1}(\tau) \text { with } i=1, \ldots, n_{r}-1, \\
T_{e} \dot{x}_{r, n_{r}}(\tau) & =\sum_{i=1}^{n_{r}} \alpha_{i} x_{r, i}(\tau)+x_{2}(\tau)-w(\tau)
\end{aligned}
$$

with $T_{e}:=\frac{2}{t_{f}-t_{0}}$. In the following, we can transform the continuous infinite dimensional optimization problem (12) into a finite dimensional NLP using the pseudospectral method and differential flatness.

We choose the collocation points $\left(\tau_{1}, \ldots, \tau_{N}\right)$ within the interior of the interval $(-1,1)$ and two noncollocated points $\tau_{0}=-1$ and $\tau_{f}=1$. The collocation points are the roots of the $N$ th order Legendre polynomial $P_{N}(\tau)$, and are called Legendre-Gauss (LG) points.

$x_{k}(\tau)$ with $k=1, \ldots, n$ are approximated by

$$
x_{k}(\tau) \approx \mathbf{x}_{k}(\tau)=\sum_{j=0}^{N} L_{j}(\tau) x_{k}\left(\tau_{j}\right)
$$

where the basis function $L_{j}(\tau)$ is the Lagrangian polynomial

$$
L_{j}(\tau)=\prod_{\substack{i=0 \\ i \neq j}}^{N} \frac{\tau-\tau_{i}}{\tau_{j}-\tau_{i}}
$$

with the property

$$
L_{j}\left(\tau_{l}\right)= \begin{cases}1 & \text { if } l=j \\ 0 & \text { if } l \neq j\end{cases}
$$

Thus we have $\mathbf{x}_{k}\left(\tau_{j}\right)=x_{k}\left(\tau_{j}\right)$ from (18) and (20). The input $u(\tau)$ can also be parameterized in a similar way with $u\left(\tau_{1}\right), \ldots, u\left(\tau_{N}\right)$ as the collocation points. (18)

The first derivative of $\mathbf{x}_{k}(\tau)$ in terms of $\tau$ at the collocation points $\tau_{i}$ can be obtained by differentiating

$$
\dot{\mathbf{x}}_{k}\left(\tau_{i}\right)=\sum_{j=0}^{N} \dot{L}_{j}\left(\tau_{i}\right) x_{k}\left(\tau_{j}\right)
$$

which can be expressed in a compact matrix form as

$$
\dot{X}_{k}=D_{0} x_{k}\left(\tau_{0}\right)+D_{N} X_{k}
$$

where $X_{k}:=\left[\begin{array}{llll}\mathbf{x}_{k}\left(\tau_{1}\right), & \mathbf{x}_{k}\left(\tau_{2}\right), & \ldots, & \mathbf{x}_{k}\left(\tau_{N}\right)\end{array}\right], D=\left[D_{0}, D_{N}\right]=\left[\dot{L}_{j}\left(\tau_{i}\right)\right] \in \mathbb{R}^{N \times(N+1)}$ with $i=1, \ldots N$ and $j=0, \ldots, N, D_{0} \in \mathbb{R}^{N \times 1}$ and $D_{N} \in \mathbb{R}^{N \times N}$. 
Using the equation (22), (17a) - (17d) can be written as

$$
\begin{aligned}
T_{e} D_{N} X_{1}= & -T_{e} D_{0} x_{1}\left(\tau_{0}\right)-X_{2}+W \\
T_{e} D_{N} X_{2}= & -T_{e} D_{0} x_{2}\left(\tau_{0}\right)+\frac{1}{m} \bar{\kappa}\left(X_{1}\right)-\frac{D_{f}}{m} X_{2}-\frac{1}{m} \sum_{i=1}^{n_{r}} \beta_{i} X_{r, i}+\frac{1}{m} U \\
X_{r, i}= & \sum_{j=1}^{i-1} T_{e}^{i-j} D_{N}^{i-j-1} D_{0} x_{r, j}(0)+T_{e}^{i-1} D_{N}^{i-1} X_{r, 1} \\
& \text { with } i=1, \ldots, n_{r}-1, \\
T_{e} D_{N} X_{r, n_{r}}= & -T_{e} D_{0} x_{r, n_{r}}\left(\tau_{0}\right)+\sum_{i=1}^{n_{r}} \alpha_{i} X_{r, i}+X_{2}-W
\end{aligned}
$$

where $\bar{\kappa}\left(X_{1}\right):=\left[\kappa\left(\mathbf{x}_{1}\left(\tau_{1}\right), \kappa\left(\mathbf{x}_{1}\left(\tau_{2}\right), \ldots, \kappa\left(\mathbf{x}_{1}\left(\tau_{N}\right)\right]^{T}, U:=\left[u\left(\tau_{1}\right), u\left(\tau_{2}\right), \ldots, u\left(\tau_{N}\right)\right]^{T}\right.\right.\right.$ and $W:=\left[w\left(\tau_{1}\right), w\left(\tau_{2}\right), \ldots\right.$, $\left.w\left(\tau_{N}\right)\right]^{T}$ comprises the wave profile $w(\tau)$ at the collocation points.

Choose $x_{1}(\tau)$ as the flat output. Then after some straightforward manipulations of equations (23a) (23d), $X_{2}$ and $U$ can be expressed by $X_{1}$, the initial states, and the wave prediction $W$ as

$$
\begin{aligned}
X_{2}= & -T_{e} D_{0} x_{1}\left(\tau_{0}\right)-T_{e} D_{N} X_{1}+W \\
U= & {\left[\sum_{i=1}^{n_{r}-1}\left(\beta_{i} I_{N}+\beta_{n_{r}} \alpha_{i} S_{a}\right) T_{e}^{i-1} D_{N}^{i-1}-T_{e}\left(\beta_{n_{r}} S_{a}+S_{b}\right) D_{N}\right] X_{1} } \\
& -\bar{\kappa}\left(X_{1}\right)-\left[S_{b} m D_{0}+\beta_{n_{r}} S_{a}\right] D_{0} x_{1}\left(\tau_{0}\right)+m T_{e} D_{0} x_{2}\left(\tau_{0}\right) \\
& +\sum_{i=1}^{n_{r}-1}\left(\beta_{i} I_{N}+\beta_{n_{r}} \alpha_{i} S_{a}\right) \sum_{j=1}^{i-1} T_{e}^{i-j} D_{N}^{i-j-1} D_{0} x_{r, j}\left(\tau_{0}\right) \\
& -\beta_{n_{r}} T_{e} S_{a} D_{0} x_{r, n_{r}}(0)+S_{b} W
\end{aligned}
$$

where $S_{a}:=\left(T_{e} D_{N}+\alpha_{n_{r}} I_{N}\right)^{-1}, S_{b}:=m T_{e} D_{N}+D_{f} I_{N}$ and identity matrix $I_{N} \in \mathbb{R}^{N \times N}$.

The objective function in (12) can be approximated by

$$
\int_{t_{0}}^{t_{f}} u(t) y(t) \approx \frac{t_{f}-t_{0}}{2} \sum_{i=1}^{N} w_{g}\left(\tau_{i}\right) u\left(\tau_{i}\right) x_{2}\left(\tau_{i}\right)=\frac{t_{f}-t_{0}}{2} X_{2}^{T} W_{g} U
$$

where $W_{g}:=\operatorname{diag}\left(w_{g}\left(\tau_{1}\right), w_{g}\left(\tau_{2}\right), \ldots, w_{g}\left(\tau_{N}\right)\right)$ is a weight matrix with

$$
w_{g}\left(\tau_{i}\right):=\frac{2}{1-\tau_{i}^{2}\left[\dot{P}_{N}\left(\tau_{i}\right)\right]^{2}} .
$$

Here $\dot{P}_{N}$ is the derivative of Legendre polynomial with degree $N$.

The constrained optimal control can thus be formulated as a NLP

$$
\begin{array}{ll}
\min _{X_{1}} & \frac{t_{f}-t_{0}}{2} X_{2}^{T} W_{g} U \\
\text { s.t. } & -U_{\max } \leq U \leq U_{\max } \\
& -Z_{\max } \leq X_{1} \leq Z_{\max }
\end{array}
$$

where $U_{\max }:=\left[u_{\max }, \ldots, u_{\max }\right]^{T} \in \mathbb{R}^{N}$, and $Z_{\max }:=\left[z_{\max }, \ldots, z_{\max }\right]^{T} \in \mathbb{R}^{N}$. Therefore, a $N$-dimmensional NLP with only $X_{1}$ as the optimization variable by substituting (23a) and (24b) into (26a) and (26b). This reduction of dimension can increase the computational speed significantly, compared with the dimension of 
$N \times n$ of the corresponding equation derived from using pseudospctral method alone. This difference is more obvious for higher order model. Note that the equality constraints for the dynamics are not needed in (26), since all the dynamic information has already been involved in the input $U$ through (24b).

If the power can only be delivered from the WEC to the grid, then $N$ extra quadratic constraints must be imposed to the NLP problem:

$$
X_{2} \odot U \succeq \mathbf{0}
$$

where $\odot$ denotes element-wise product, $\succeq$ denotes element-wise inequality, and $\mathbf{0}$ is a $n \times 1$ zero vector.

\subsection{Linear model case}

If the WEC model is linear, then a quadratic programming (QP) problem can be derived. In this case study, the linear WEC model can be derived by assuming the float is a cylinder with a constant radius $r_{1}$, so that the stiffness is a constant $K$. With this assumption, the equation (24b) can be written concisely as

$$
U=M_{x} X_{1}+M_{0} x\left(\tau_{0}\right)+S_{b} W
$$

where $M_{x}$ and $M_{0}$ can be derived from (24b) straightforwardly after replacing $\bar{\kappa}\left(X_{1}\right)$ by $K X_{1}$.

Substituting (28) into the NLP (26), we have a QP

$$
\begin{array}{ll}
\min _{X_{1}} & \frac{1}{2} X_{1}^{T} H X_{1}+X_{1}^{T} F \\
\text { s.t. } & -U_{\max } \leq U \leq U_{\max } \\
& -Z_{\max } \leq X_{1} \leq Z_{\max }
\end{array}
$$

with

$$
\begin{aligned}
H & :=\mathbf{H e}\left(T_{e} D_{N}^{T} W_{g} M_{x}\right) \\
F & =M_{x}^{T} W_{g}\left(W-T_{e} D_{0} x_{1}\left(\tau_{0}\right)\right)-T_{e} D_{N}^{T} W_{g}\left(M_{0} x\left(\tau_{0}\right)+S_{b} W\right)
\end{aligned}
$$

Here $\operatorname{He}(M):=M+M^{T}$ for a square matrix $M$.

\subsection{Online implementation of $N M P C$}

The optimization method developed in this section needs to be implemented online at each sampling instant in a receding horizon manner. The necessity of implementing the optimization online instead of offline comes from the following concerns: first incoming wave profile changes constantly, which needs to be predicted online by a separate wave prediction algorithm; second, online implementation introduces feedback mechanism, which helps strengthen the inherent robustness of the WEC control system. The online implementation is realized in the following steps:

Step 1: At sampling instant $k$, the wave profile $W$ is predicted. The optimization (26) (or (29) for linear case) is resolved.

Step 2: An optimal control sequence $U^{*}$ is calculated using (24b) (or (28) for linear case). The first element of the sequence, $u^{*}\left(\tau_{1}\right)$ is implemented as the control input for a sampling period.

Step 3: At the next sampling instant $k+1$, the procedure repeats.

\section{Numerical simulation}

The numerical parameters of the WEC are mostly adopted from those used in [15], and are summarized in Table 1. Note that the parameters do not reflect the real parameters used in PB150, and they are mainly used for demonstration purpose. The frequency dependent added damping is

$$
\hat{D}(j \omega)=\frac{1.5 \times 10^{4} \times(j \omega+0.01)(j \omega+0.02)}{(j \omega+0.1)(j \omega+0.2)^{2}}
$$


Table 1: Parameters used for the WEC model

\begin{tabular}{lll} 
Description & Notation & Values \\
Density of sea water & $\rho$ & $1025 \mathrm{~kg} / \mathrm{m}^{3}$ \\
Gravity & $g$ & $9.8 \mathrm{~N} / \mathrm{kg}$ \\
Float radius & $r$ & $4.5 \mathrm{~m}$ \\
Damping (friction) & $D_{f}$ & $2 \times 10^{3} \mathrm{Nm} / \mathrm{s}$ \\
Float mass & $m_{s}$ & $1 \times 10^{4} \mathrm{~kg}$ \\
Added mass & $m_{a}$ & $7 \times 10^{4} \mathrm{~kg}$ \\
Total mass & $m$ & $8 \times 10^{4} \mathrm{~kg}$ \\
Stiffness & $K$ & $6.39 \times 10^{5} \mathrm{~N} / \mathrm{m}$ \\
Input force limit & $u_{\max }$ & $3 \times 10^{5} \mathrm{~N}$ \\
Float heave limit & $h_{1}$ & $1.2 \mathrm{~m}$ \\
\hline
\end{tabular}
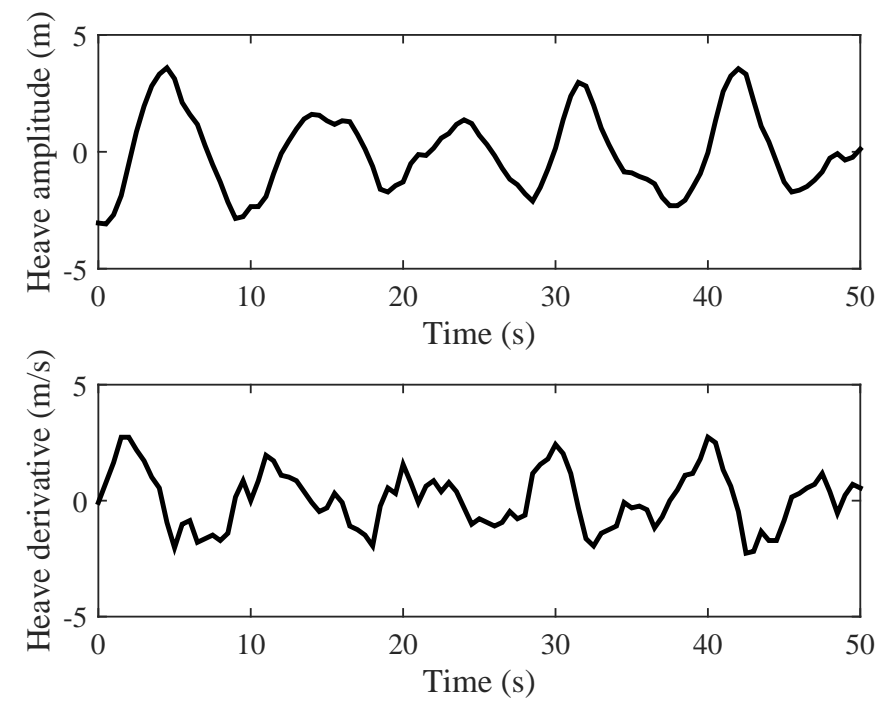

Figure 4: A 50 seconds period of wave profile (wave elevation and its derivative) gathered off the coast of Cornwall, England is used in simulations. 


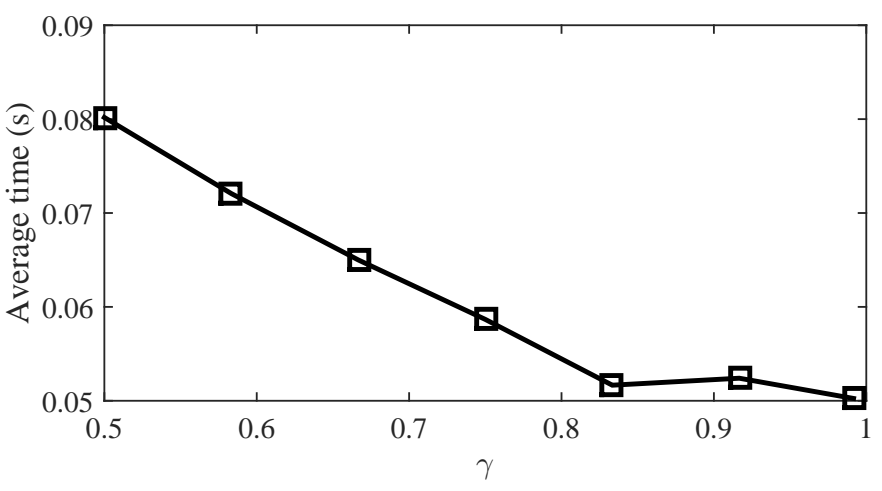

Figure 5: Average computational time versus $\gamma=h_{2} / h_{1}$.

Its maximum damping coefficient is $90 \mathrm{~dB}$ at $0.2 \mathrm{rad} / \mathrm{sec}$; this roughly matches the magnitude of a constant damping coefficient term $D=2 \times 10^{5} \mathrm{Nm} / \mathrm{s}$ in the second order model used in [14]. The order of the model used in the simulation is $n=5$. A real wave profile of 50 seconds, gathered off the coast of Cornwall, UK is used. Its heave motion amplitude and its first derivative are shown in Figure 4.

The WEC constrained optimal control problem is formulated using the approach proposed in section 3 . The numerical simulations are run on a PC with Intel ${ }^{\circledR} \mathrm{Core}^{\mathrm{TM}} \mathrm{i} 7 \mathrm{CPU}$ at $2.10 \mathrm{GHz}$. At each time instant $k$, a NLP is resolved by the sequential quadratic programming (SQP) method using the MATLAB ${ }^{\mathrm{TM}}$ routines fmincon for the nonlinear model or quadprog for the linear model.

Two scenarios about energy flow direction are simulated. In the bidirectional energy flow scenario, the power generated from the WEC can be delivered to the grid; or conversely, the power from the grid can flow back to the WEC. One of the possible realization of this bidirectional power flow is reported in [? ]. In the unidirectional energy flow scenario, the energy can only be generated by the WEC and delivered to the grid. Compared with the bidirectional flow, the unidirectional flow design can be less costly and easier to achieve; however, its energy output can be smaller for an extra constraint on energy flow $P(t) \geq 0$ is imposed on the optimization.

The control input constraint is $|u| \leq u_{\max }$ with $u_{\max }=3 \times 10^{5} \mathrm{~N}$; the constraint on the difference between the heave motion of the float and the elevation of the water is $|z| \leq z_{\max }$ with $z_{\max }=h_{1}=1.2 \mathrm{~m}$. The dimensions of the float in Figure 2 are $r_{1}=4.5 \mathrm{~m}, r_{2}=2 \mathrm{~m}, h_{1}=1.2 \mathrm{~m}$ and $h_{2}$ is chosen as different values for testing. The power flow is assumed to be bidirectional.

To show the influence of the nonlinear effect on the computational time, we use the ratio $\gamma=h_{2} / h_{1}$ to indicate the degree of the nonlinear effect of the hydrostatic stiffness. The nonlinear effect diminishes when $\gamma \rightarrow 1$. By fixing the number of collocation points $N=10$, the prediction horizon $t_{f}=2 \mathrm{~s}, h_{1}=1.2 \mathrm{~m}$ and changing $h_{2}$, we plot the average computational time per optimization versus the ratio $\gamma$ in Figure 5 . It shows that the computational burden decreases dramatically with the degree of the nonlinear effect when $\gamma$ is small and this tendency becomes moderate when $\gamma \rightarrow 1$. If the float is assumed to be a cylinder with constant radius, i.e. $r_{1}=r_{2}=4.5 \mathrm{~m}$ and $h_{1}=h_{2}=1.2 \mathrm{~m}$, then the hydrostatic stiffness is a constant value, $K=\rho g h_{1} \pi r_{1}^{2}=6.39 \times 10^{5} \mathrm{~N} / \mathrm{m}$. In this case, the WEC model becomes a linear model and the quadratic programming can be used to resolve the problem. For the case of $N=10$, the average computational time per optimization is $0.03 \mathrm{~s}$ for solving a QP. Due to the alleviated computational burden using a QP, it is preferable to consider using a MPC based on a linear model to control a WEC if its nonlinearities are trivial. However, for the plant with significant nonlinearities, using the linear MPC can cause performance degradation, in terms of energy output reduction and constraint violation due to the model mismatch.

Next we demonstrate the influence of the number of collocation points on the average computational time and power output by fixing $h_{2}=1.0 \mathrm{~m}$, which corresponds to a moderate nonlinear effect. Figure 6 shows that the average computational time per optimization increases linearly with the number of collocation points. Figure 7 shows that the average power output increases with the collocation points dramatically 


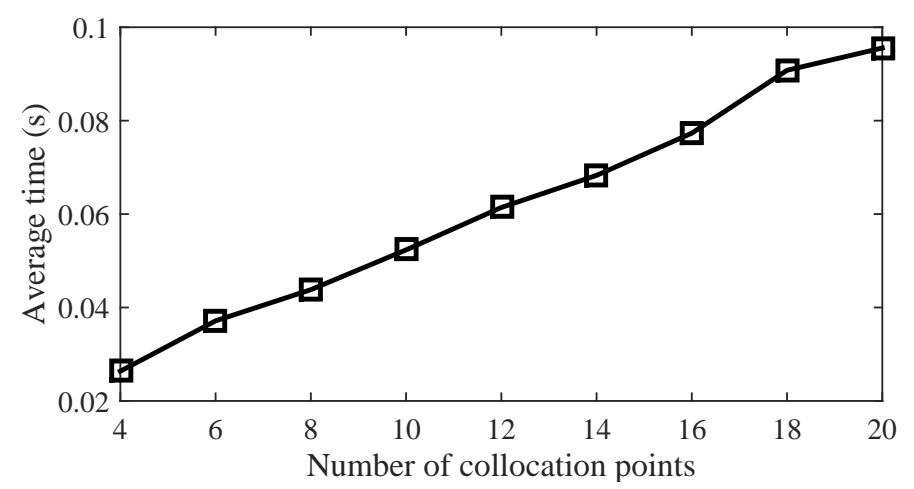

Figure 6: Average computational time versus number of collocation points $\left(h_{1}=1.2 \mathrm{~m}, h_{2}=1.0 \mathrm{~m}, t_{f}=2.0 \mathrm{~s}\right)$.

when $N \leq 10$, and the average power output starts oscillating after $N>14$. This means increase of the collocation points beyond 14 cannot obviously further improve energy output. Considering the ever growing computational burden with $N$ from Figure 6 , the benefit of choosing a very big $N$ (e.g. 20) for larger energy output is compromised by the added computational burden. Thus for this simulation scenario, a choice of $N=10 \sim 14$ is a reasonable tradeoff. For other scenarios, the value of $N$ can be changing and needs to be selected carefully after a series of simulations.

To demonstrate the effectiveness of handling constraints, we only show one case where $N=10, t_{f}=2$ $\mathrm{s}, h_{2}=1.0 \mathrm{~m}$. The power constraint is also imposed by assuming a one directional flow from the WEC to the grid, i.e. $P(t) \geq 0$. Figure 8 shows that all the constraints are strictly satisfied with the presence of active constraints. The time average power output with the constraint $P(t) \geq 0$ is $\bar{P}=307 \mathrm{~kW}$, which is approximately a $5 \%$ reduction of energy generation compared to the case of bidirectional power flow $(\bar{P}=323 \mathrm{~kW})$. Since the bidirectional design can be relatively more costly, it might be interesting to evaluate the tradeoff between the bidirectional and unidirectional designs when using the control strategy proposed in this paper in the future work, which is beyond the scope of this paper.

Finally, we have the following remarks. The average computational time for solving one optimization using the approach based on flatness is fast enough for online MPC implementation purposes. The benefit of using this method is significant for high order and nonlinear WEC model. Since the average computational time varies when different computers and different optimization software packages are used, the simulation in this paper is mainly for the purpose of demonstrating the effectiveness of the flatness approach. For the real time application, the computational time is supposed to be even much faster if the optimization algorithm can be programmed in C specifically for the WEC control problem rather than using the generic optimization routines in MATLAB.

\section{Conclusion}

An efficient optimization approach based on the pseudospectral method exploiting the flatness property is developed for nonlinear model predictive control of a point absorber. This approach is more efficient than the optimization based on pseudospectral method alone, while still enjoys the benefits of coping with more complicated optimization problem than quadratic programming. In this paper, we demonstrate its efficacy for NMPC of a typical point absorber described by a nonlinear model, with non-convex objective function and nonlinear constraints. The nonlinearity comes from the buoyancy force due to non-constant radius of the float. The method developed in this paper can be potentially extended to other nonlinear WEC models in a straightforward manner. 


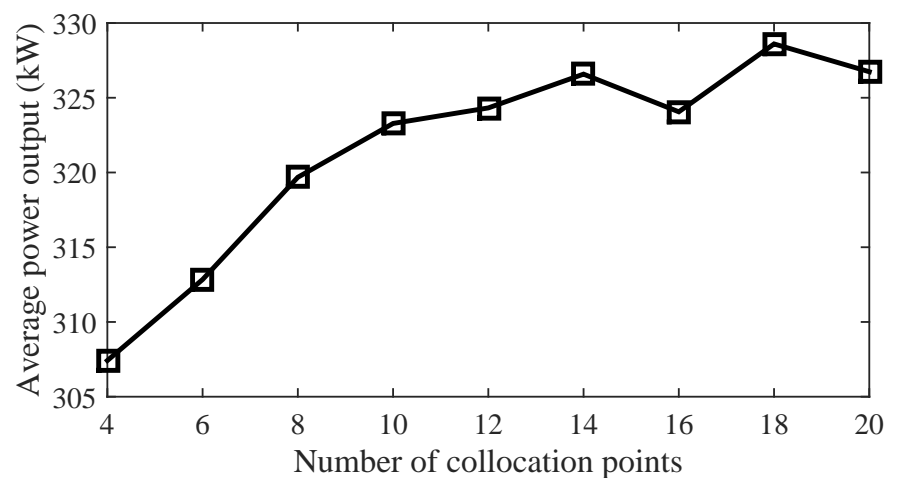

Figure 7: Average power output versus number of collocation points $\left(h_{1}=1.2 \mathrm{~m}, h_{2}=1.0 \mathrm{~m}, t_{f}=2.0 \mathrm{~s}\right)$.

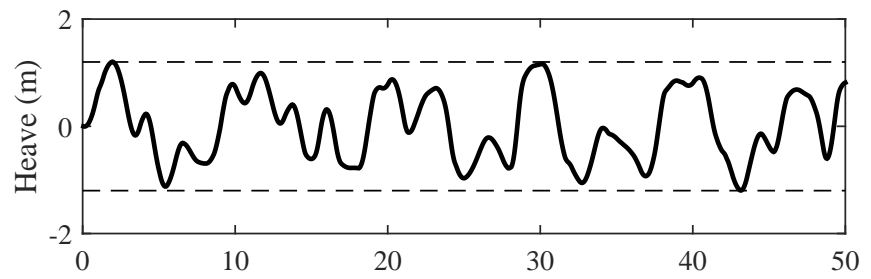

(a) Simulation time (s)

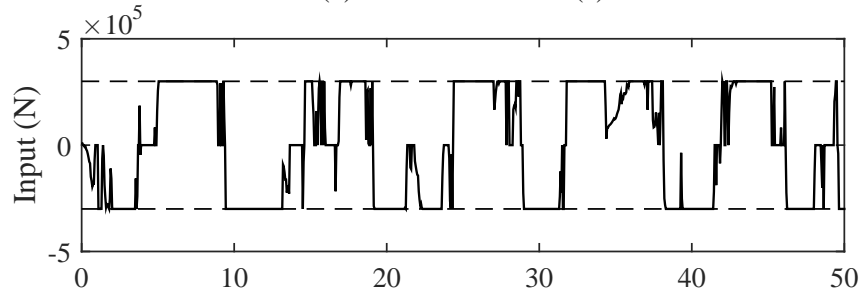

(b) Simulation time (s)

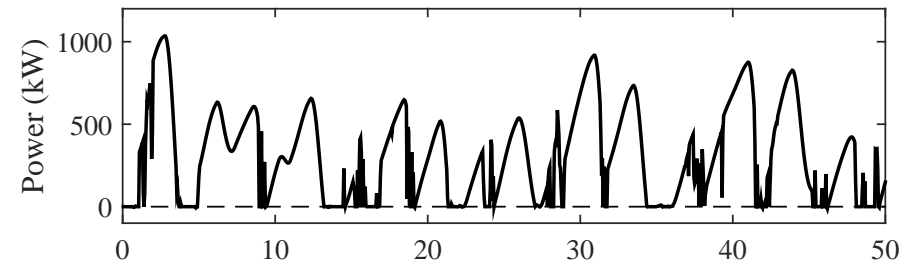

(c) Simulation time (s)

Figure 8: (a) Difference between float heave and water elevation, $x_{1}\left(z_{\max }=1.2 \mathrm{~m}\right)$; (b) Control input, $\mathrm{u}\left(u_{\max }=3 \times 10^{5} \mathrm{~N}\right)$; (c) Power output $(P \geq 0)$. 


\section{Bibliography}

[1] K. Budal, J. Falnes, Optimum operation of improved wave-power converter, Marine Science Communciations 3 (2) (1977) $133-150$.

[2] K. Budal, J. Falnes, T. Hals, L. C. Iversen, T. Onshus, Model experiment with a phase controlled point absorber, in: Proc. of Second Int. Symp. on Wave and Tidal Energy, Cambridge, UK, 1981, pp. 191-206.

[3] K. Budal, J. Falnes, L. C. Iversen, P. Lillebekken, G. Oltedal, T. Hals, T. Onshus, A. Høy, The Norwegian wave-power buoy project, Tapir, Trondheim, 1982, pp. 323-344.

[4] G. A. Nolan, J. V. Ringwood, W. E. Leithead, S. Butler, Optimal damping profiles for a heaving buoy wave energy converter, in: The Fifteenth Int. Offshore and Polar Eng. Conf., Seoul, South Korea, 2005, pp. 477-484.

[5] H. Eidsmoen, On theory and simulation of heaving buoy wave energy converters with control, Ph.D. thesis, Norwegian University of Science and Technology, Trondheim (1996).

[6] M. Greenhow, S. P. White, Optimal heave motion of some axisymmetric wave energy devices in sinusoidal waves, Applied Ocean Research 17 (1997) 141-159.

[7] J. Falnes, Ocean Waves and Oscillating Systems, Cambridge University Press, 2002.

[8] U. A. Korde, Latching control of deep water wave energy devices using an active references, Ocean Engineering 29 (11) (2002) 1343-1355.

[9] A. Babarit, A. H. Clément, Optimal latching control of a wave energy device in regular and irregular waves, Applied Ocean Research 28 (2006) 79-91.

[10] F. Fusco, J. V. Ringwood, Linear models for short-term wave forecasting, in: Proc. World Renewable Energy Conference, Glasgow, UK, 2008.

[11] T. K. A. Brekken, On model predictive control for a point absorber wave energy converter, in: PowerTech, 2011 IEEE Trondheim, 2011, pp. 1-8.

[12] J. A. M. Cretel, G. Lightbody, G. P. Thomas, A. W. Lewis, Maximisation of energy capture by a wave-energy point absorber using model predictive control, in: Proc. 18th IFAC World Congress, Milano, 2011.

[13] J. Hals, J. Falnes, T. Moan, Constrained optimal control of a heaving buoy wave-energy converter, J. of Offshore Mechanics and Arctic Engineering 133 (1) (2011) 011401.

[14] G. Li, G. Weiss, M. Mueller, S. Townley, M. B. Belmont, Wave energy converter control by wave prediction and dynamic programming, Renewable Energy 48 (2012) 392-403.

[15] G. Li, M. R. Belmont, Model predictive control of sea wave energy converters-Part I: A convex approach for the case of a single device, Renewable Energy 69 (2014) 453-463.

[16] E. Abraham, E. Kerrigan, Optimal active control and optimization of a wave energy converter, Sustainable Energy, IEEE Transactions on $4(2)$.

[17] G. Li, M. R. Belmont, Model predictive control of sea wave energy converters-Part II: The case of an array of devices, Renewable Energy 68 (2014) 540-549.

[18] M. Richter, M. Magana, O. Sawodny, T. Brekken, Nonlinear model predictive control of a point absorber wave energy converter, Sustainable Energy, IEEE Transactions on 4 (1) (2013) 118-126.

[19] N. Tom, R. W. Yeung, Nonlinear model predictive control applied to a generic ocean-wave energy extractor, J. Offshore Mech. Arct. Eng. 136 (4).

[20] D. Benson, A Gauss pseudospectral transcription for optimal control, Ph.D. thesis, Massachussetts Institute of Technology (2005).

[21] G. Elnagar, M. Kazemi, M. Razzaghi, The pseudospectral legendre method for discretizing optimal control problems, Automatic Control, IEEE Transactions on 40 (10) (1995) 1793-1796.

[22] D. Garg, M. Patterson, W. W. Hager, A. V. Rao, D. A. Benson, G. T. Huntington, A unified framework for the numerical solution of optimal control problems using pseudospectral methods, Automatica 46 (11) (2010) 1843 - 1851.

[23] Q. Gong, W. Kang, N. Bedrossian, F. Fahroo, P. Sekhavat, K. Bollino, Pseudospectral optimal control for military and industrial applications, in: Decision and Control, 2007 46th IEEE Conference on, 2007, pp. 4128-4142.

[24] D. R. Herber, J. T. Allison, Wave energy extraction maximization in irregular ocean waves using pseudospectral methods, in: Proceedings of the ASME International Design Engineering Technical Conferences \& Computers and Information in Engineering Conference, Portland, Oregon, USA, 2013, pp. 1817-1824.

[25] M. Fliess, J. Lévine, P. Martin, P. Rouchon, Flatness and defect of non-linear systems: introductory theory and examples, International Journal of Control 61 (6) (1995) 1327-1361.

[26] M. Fliess, R. Marquez, Continuous-time linear predictive control and flatness: A module-theoretic setting with examples, International Journal of Control 73 (7) (2000) 606-623.

[27] T. Meurer, Flatness-based trajectory planning for diffusionreaction systems in a parallelepipedona spectral approach, Automatica 47 (5) (2011) 935 - 949.

[28] R. Mahadevan, F. J. Doyle III, Efficient optimization approaches to nonlinear model predictive control, International Journal of Robust and Nonlinear Control 13 (3-4) (2003) 309-329.

[29] J. Oldenburg, W. Marquardt, Flatness and higher order differential model representations in dynamic optimization, Computers \& Chemical Engineering 26 (3) (2002) $385-400$.

[30] D. Schwarzmann, J. Lunze, R. Nitsche, A flatness-based approach to internal model control, in: American Control Conference, 2006.

[31] G. Weiss, G. Li, M. Mueller, S. Townley, , M. R. Belmont, Optimal control of wave energy converters using deterministic sea wave prediction, in: The Energy \& Materials Research Conference, Torremolinos, Malaga, Spain, 2012. 
[32] Q.-C. Zhong, G. Weiss, Synchronverters: Inverters that mimic synchronous generators, IEEE Trans. on Industrial Electronics 58 (2011) 1259-1267.

[33] M. R. Belmont, Increases in the average power output of wave energy converters using quiescent period predictive control, Renewable Energy 35 (2010) 2812-2820. 